\title{
REVISTAMATERIA
}

\section{Dureza Brinell y parámetros termofísicos de la Guadua angustifólia - Kunth}

\section{Brinell hardness and thermophysical parameters of the Guadua angustifolia - Kunth}

\author{
Gordillo-Delgado, F. ${ }^{1,2}$, Cortés-Hernández, D. M. ${ }^{1}$, \\ Mejía-Morales, C. ${ }^{1}$, Bedoya-Pérez, A.F. ${ }^{1}$, Ariza-Calderón, H. ${ }^{1}$.
}

\author{
${ }^{1}$ Laboratorio de Optoelectrónica, Universidad del Quindío. \\ Apdo. Postal 2639 Armenia, Colombia. \\ e-mail: dmchernandez@hotmail.com ; clami870611@hotmail.com ; heariza@uniquindio.edu.co ; \\ adrian_filipo@hotmail.com \\ ${ }^{2}$ Centro de Investigación en Ciencia aplicada y Tecnología Avanzada \\ del I.P.N, Unidad Legaria. Av. Legaria 694. Col. Irrigación, México D.F \\ e-mail: fgordillo@uniquindio.edu.co
}

\section{RESUMEN}

La Guadua angustifolia-Kunth es un recurso forestal que debido a sus características físicas y mecánicas ha sido utilizado principalmente en la construcción de viviendas, en la fabricación de laminados y de artículos decorativos. Además es una planta que desempeña un importante papel como protectora de cuencas hidrográficas, en la captura de $\mathrm{CO}_{2}$ y en la conservación de suelos. La caracterización térmica y mecánica de este tipo de materiales de origen biológico es de mucha importancia ya que permiten optimizar los procesos de transformación industrial para mejorar la calidad del producto final. En este trabajo se reportan mediciones de difusividad térmica, efusividad térmica y dureza Brinell (DB) que fueron hechas a muestras tomadas a lo largo del culmo de la guadua, considerando las zonas conocidas como cepa, basa y sobrebasa. Los parámetros termofísicos fueron medidos haciendo uso de la técnica fotoacústica mientras que los valores de DB fueron calculados de acuerdo con estándares internacionales. Los resultados muestran que las magnitudes asociadas al transporte de calor y a la deformación superficial en la guadua son homogéneas a lo largo de ella. Se comprobó que este comportamiento presenta alta correlación con las características morfoanatómicas de la planta, que fueron determinadas a través de la observación de micrografías.

Palavras-chave: Guadua angustifolia - Kunth, difusividad térmica, efusividad térmica, dureza Brinell.

\section{ABSTRACT}

Guadua angustifolia-Kunth is a forest resource with special physical and mechanical characteristics, which is used mainly in houses building, laminates manufacture, and decorative items. Also it plays an important role in watershed protection, in the $\mathrm{CO} 2$ capture and soil conservation. The thermal and mechanical characterization of this type of biological materials is very important for optimizing the industrial transformation processes and improving the final product quality. In this work, thermal diffusivity, thermal effusivity and Brinell hardness (DB) of bottom, middle and top culm areas along the Guadua were considered. These thermo-physical parameters were measured using photoacoustic technique, while DB values were calculated according to the international standards. Results show that heat transport and surface deformation magnitudes are homogeneous along this bamboo. It was found that this behavior is highly correlated with the morpho-anatomical characteristics of the plant, which were determined through photo-micrographs.

Keywords: Guadua angustifolia - Kunth, thermal diffusivity, thermal effusivity, Brinell hardness. 


\section{INTRODUCCIÓN}

La Guadua angustifolia-Kunth es un recurso renovable de rápido crecimiento y de fácil cultivo. Es un material más liviano que la madera y mecánicamente más resistente, por lo que puede sustituirla en algunos casos [1]. Este bambú ha sido tradicionalmente empleado en el campo de la construcción y en la fabricación de artículos decorativos [2, 3]. Además, ofrece diversos beneficios ambientales ya que actúa como regulador de agua de los cauces de los ríos, son sumideros de carbono, producen oxígeno, y evitan la erosión del suelo [4]. Debido al interés que ha adquirido este recurso para la industria es conveniente llevar a cabo estudios locales sobre sus características mecánicas y térmicas que permitan mejorar los procesos de secado y de transformación.

En este trabajo se muestran mediciones de difusividad térmica ( $\alpha$ ), efusividad térmica (e) y dureza Brinell (DB) hechas a muestras de Guadua angustifolia Kunth. Los dos primeros parámetros se obtuvieron para cuatro regiones radiales de la sección transversal a lo largo del culmo dividido en tramos correspondientes a la cepa, basa y sobrebasa. Los valores de DB para la región externa de la guadua se adquirieron de acuerdo con los estándares planteados en las normas DIN EN 1534. Ya que las propiedades térmicas y estructurales de un material están ligadas a sus características morfoanatómicas, se analizó el porcentaje de fibras, parénquima y haces vasculares en las direcciones longitudinal y radial, con el fin de encontrar relaciones entre las variables consideradas.

Los parámetros termofísicos miden efectos de la aplicación de temperatura a un cuerpo y son de especial interés en el campo de la transformación y de la conservación de materiales de origen biológico [5]. Una técnica no destructiva que permite el cálculo de estos parámetros es la técnica fotoacústica (FA). El efecto FA es producido cuando un haz de luz modulado periódicamente incide sobre una muestra generando calentamiento por procesos de desexcitación no radiativos; este calentamiento genera cambios de presión en la capa de gas adyacente a la muestra, los cuales son detectados por un micrófono [6]. La señal FA está relacionada con el transporte de calor a través del material de acuerdo con el modelo de A. Rosencwaig y A. Gersho [7].

La DB se obtiene por un método de indentación en el que se mide la penetración de un objeto en el material a estudiar. Este método es ampliamente usado para probar metales y no metales de dureza baja y media [8, 9]. Una buena dureza significa generalmente que el material es resistente al rayado y al desgaste [10].

\section{MATERIALES Y MÉTODOS}

Se utilizaron 19 guaduas variedad castilla con edad promedio de 5 años, cultivadas en el municipio de Caicedonia (Valle del Cauca) a una altura de $1100 \mathrm{msnm}$. Las plantas se cortaron en tramos de $4 \mathrm{~m}$ y se identificaron según la posición a lo largo del tramo (cepa, basa y sobrebasa). Para los ensayos de dureza Brinell se empleó la norma Europea DIN EN 1534, que recomienda dimensiones de muestra de 40x40x5mm. Se utilizó una esfera metálica o indentador con diámetro de $10 \mathrm{~mm}$ y una fuerza de $1000 \mathrm{~N}$ perpendicular a la superficie de la muestra. Se llevaron a cabo ensayos en cinco zonas diferentes con el fin de obtener un promedio para cada muestra en particular y se hizo la medición del diámetro de cada huella en la muestra en forma paralela y perpendicular a la fibra para obtener dos medidas por cada huella y un total de 10 medidas por cada muestra. El contenido de humedad de las muestras en el momento de realizar los ensayos fue de 14 $\pm 2 \%$.

Para obtener la difusividad y efusividad térmica de cada muestra se dividió la sección transversal en cuatro partes, denominando 1 a la interna y 4 a la externa que incluye la corteza. Las muestras se adelgazaron hasta alcanzar aproximadamente $600 \mu \mathrm{m}$. La técnica FA resuelta en frecuencia, cuyo montaje es descrito en 
la Figura 1, fue utilizada para hacer las estimaciones de difusividad térmica considerando como variables zonas radiales y longitudinales de la guadua. En el montaje experimental, un haz de luz proveniente de un láser de Argón de longitud de onda de $488 \mathrm{~nm}$ es pulsado mediante un modulador acusto-óptico. Este se hace incidir sobre la muestra colocada en una celda FA abierta. En este tipo de celda la muestra queda en contacto directo con la cubierta de un micrófono de tal forma que sella su cámara interna. Para las medidas de efusividad térmica se empleó una celda FA cerrada y una lámina de plata con espesor de $210 \mu \mathrm{m}$ como material absorbedor.

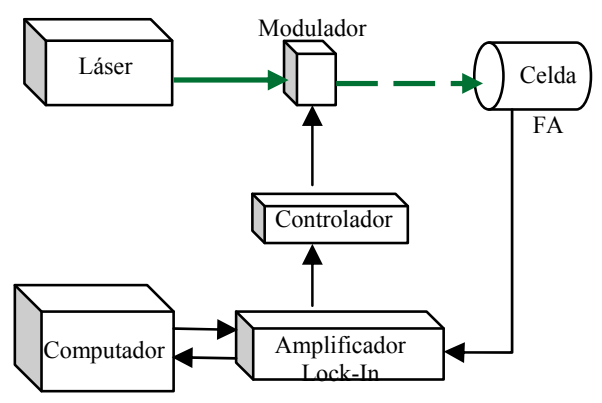

Figura 1: Diagrama de bloques que representa el montaje de la técnica FA resuelta en frecuencia. El haz de luz proveniente de un láser es modulado y luego dirigido hacia la muestra ubicada en la celda FA. El calor generado produce cambios de presión en el gas adyacente a la muestra; los cambios de presión son detectados por un micrófono, amplificados por un Lock-in y finalmente visualizados a través de un computador.

Para el análisis microscópico se cortaron pedazos de guadua de $1 \times 1 \mathrm{~cm}$, luego se adelgazaron hasta alcanzar un espesor de aproximadamente $600 \mu \mathrm{m}$ mediante cortes con un bisturí. Las microfotografías se tomaron a planos de cortes transversales y longitudinales, y se cuantificó el porcentaje de área ocupada por las fibras y los haces vasculares. En el primer caso se hizo adicionalmente el conteo de células del parénquima. Este procedimiento se llevó a cabo para cada una de las regiones, considerando la posición a lo largo del tramo, utilizando un microscopio óptico Olympus con una amplificación de 4X.

\section{RESULTADOS Y DISCUSIÓN}

Para los ensayos de dureza Brinell se empleó la ecuación (1) que establece la relación entre la dureza y la huella dejada en la superficie del material durante los ensayos. En la Figura 2 se muestra un esquema del sistema empleado.

$$
\mathrm{DB}=\frac{2 \mathrm{~F}}{\pi \mathrm{D}\left(\mathrm{D}-\sqrt{\mathrm{D}^{2}-\mathrm{d}^{2}}\right)}
$$

En esta expresión, DB es la Dureza Brinell; F es la fuerza aplicada sobre el material; D es el diámetro del indentador y d es el diámetro de la huella [8-10]. 


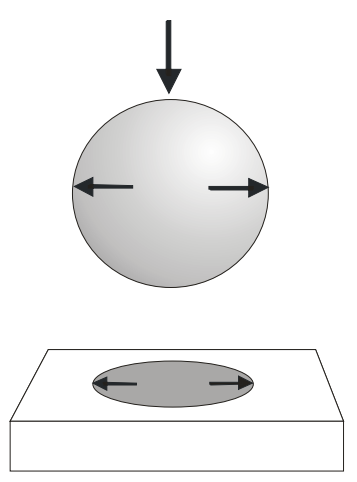

Figura 2: Esquema básico para el ensayo de dureza Brinell.

En la Tabla 1 se muestran los resultados de DB para cada una de las porciones de la guadua. Del análisis de varianza ANOVA (con un nivel de significación del 1\%) aplicado a estos datos se encontró que no existen diferencias significativas entre los valores de DB obtenidos.

Tabla 1: Valores promedio del número de dureza Brinell a lo largo del tramo.

\begin{tabular}{c|c|c|c}
\hline \multirow{2}{*}{} & \multicolumn{3}{|c}{ Número de Dureza Brinell } \\
\cline { 2 - 4 } & CEPA & BASA & SOBREBASA \\
\hline VALOR & $4,59 \pm 0,51$ & $4,59 \pm 0,41$ & $4,79 \pm 0,51$ \\
\hline
\end{tabular}

Para determinar los valores de difusividad y efusividad térmica, las curvas de intensidad de la señal FA en función de la frecuencia de modulación de la luz incidente se ajustaron por medio del modelo propuesto por A. Rosencwaig y A. Gersho para materiales ópticamente opacos y térmicamente gruesos [7].

$$
S(f)=A \frac{e^{\sqrt{f / f_{c}}}}{f}
$$

En la ecuación 2, S(f) es la amplitud de la señal FA; f es la frecuencia de modulación; A es una constante relacionada con la presión y con la temperatura ambiente, las características ópticas y térmicas del gas y la intensidad del haz incidente; y fc es la frecuencia de corte relacionada con la difusividad térmica. A esta frecuencia la longitud de difusión térmica se hace igual al espesor de la muestra. Para calcular la efusividad térmica se empleó la ecuación $3[7,11]$ :

$$
\frac{\theta}{\theta_{0}}=\frac{1}{1+\frac{b}{1_{0} \sigma_{0}}}
$$

Donde $\theta_{0}$ y $\theta$ son las amplitudes de la señal FA del material con efusividad térmica conocida y del sistema formado por la muestra unida a ésta, respectivamente; $b=e_{s} / e_{0}$ establece la razón entre la efusividad térmica del material de referencia $\left(\mathrm{e}_{0}\right)$ y de la muestra $\left(\mathrm{e}_{\mathrm{s}}\right) ; 1_{0}$ es el espesor del material de referencia y $\sigma_{0}$ es el coeficiente complejo de difusión.

En la Figura 3 aparece la gráfica de la amplitud de la señal FA en función de la frecuencia de modulación (f) para una muestra tomada de una de las guaduas y del tramo correspondiente a la basa. A partir del ajuste de esta curva, empleando la ecuación 2, se obtuvieron los valores de difusividad térmica en la dirección longitudinal y transversal de la guadua. En la Tabla 2 se relacionan los datos obtenidos que corresponden al promedio de 8 guaduas. Los davalores de $\alpha$ en las direcciones analizadas evidencian el comportamiento homogéneo de este parámetro a lo largo de la guadua. 


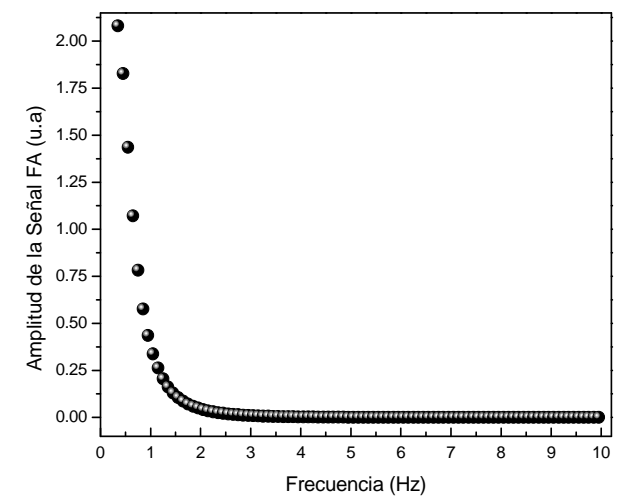

Figura 3: Amplitud de la señal FA en función de la frecuencia de modulación. En este caso la muestra utilizada proviene de la guadua etiquetada con el número $14 \mathrm{y}$ del tramo correspondiente a la basa.

Considerando el comportamiento de la difusividad térmica a lo largo del tramo, se estimó la efusividad térmica para una sola muestra escogida al azar. Para esto se ajustó la curva de la razón entre la señal FA obtenida para el sistema compuesto por la muestra de guadua unida a una delgada lámina de plata y la correspondiente a esta misma lámina, en función de la frecuencia [12]. De esta forma se obtuvo un valor de $\mathrm{e}_{\mathrm{s}}=(1000 \pm 20) \mathrm{Ws}^{1 / 2} \mathrm{~m}^{-2} \mathrm{~K}^{-1}$.

Tabla 2: Valores obtenidos de difusividad térmica y porcentaje de área ocupado por: haces vasculares (HV), fibras (F) y parénquima $(\mathrm{P})$, para muestras tomadas en las secciones transversales y longitudinales del culmo previamente definidas.

\begin{tabular}{|c|c|c|c|c|}
\hline & REGIÓN & CEPA & BASA & SOBREBASA \\
\hline \multirow{4}{*}{$\begin{array}{l}\text { DIFUSIVIDAD TÉRMICA (A) } \\
\qquad\left(10^{-6} \mathrm{M}^{2} \mathrm{~S}^{-1}\right)\end{array}$} & 1 & $0,11 \pm 0,02$ & $0,10 \pm 0,02$ & $0,12 \pm 0,03$ \\
\hline & 2 & $0,11 \pm 0,02$ & $0,12 \pm 0,02$ & $0,10 \pm 0,02$ \\
\hline & 3 & $0,11 \pm 0,01$ & $0,11 \pm 0,03$ & $0,12 \pm 0,03$ \\
\hline & 4 & $0,10 \pm 0,03$ & $0,10 \pm 0,03$ & $0,08 \pm 0,02$ \\
\hline \multirow{4}{*}{ HV (\%) } & 1 & $11 \pm 2$ & $12 \pm 3$ & $14 \pm 3$ \\
\hline & 2 & $9 \pm 2$ & $10 \pm 2$ & $11 \pm 3$ \\
\hline & 3 & $6 \pm 2$ & $7 \pm 3$ & $8 \pm 3$ \\
\hline & 4 & $3 \pm 2$ & $4 \pm 1$ & $4 \pm 1$ \\
\hline \multirow{4}{*}{$F(\%)$} & 1 & $29 \pm 6$ & $36 \pm 4$ & $32 \pm 3$ \\
\hline & 2 & $38 \pm 3$ & $46 \pm 4$ & $42 \pm 6$ \\
\hline & 3 & $58 \pm 6$ & $67 \pm 9$ & $60 \pm 5$ \\
\hline & 4 & $69 \pm 4$ & $78 \pm 7$ & $82 \pm 9$ \\
\hline \multirow{4}{*}{$P(\%)$} & 1 & $64 \pm 6$ & $52 \pm 4$ & $54 \pm 4$ \\
\hline & 2 & $53 \pm 5$ & $44 \pm 3$ & $50 \pm 2$ \\
\hline & 3 & $39 \pm 5$ & $30 \pm 9$ & $37 \pm 6$ \\
\hline & 4 & $28 \pm 3$ & $18 \pm 7$ & $14 \pm 6$ \\
\hline
\end{tabular}

El estudio morfoanatómico se hizo a través de microfotografías; se midió el porcentaje de área ocupado por los haces vasculares, parénquima y fibras a lo largo del tramo y en dirección transversal. Las muestras de guadua se adelgazaron hasta alcanzar valores alrededor de $600 \mu \mathrm{m}$ de espesor a través de cortes transversales. Una vez obtenidas las muestras, se tomaron microfotografías empleando un microscopio óptico con amplificación de 4X. Finalmente, las microfotografías se analizaron a través del programa Motic Image Plus 2.0 con el fin de calcular la razón entre el área total ocupada por estructuras de la misma clase $\left(\mathrm{A}_{\mathrm{i}}\right)$ sobre el área total de la microfotografía $\left(\mathrm{A}_{\mathrm{T}}\right)$, el valor obtenido corresponde al porcentaje de área ocupado por las estructuras. En la Tabla 2 se muestran los resultados. 


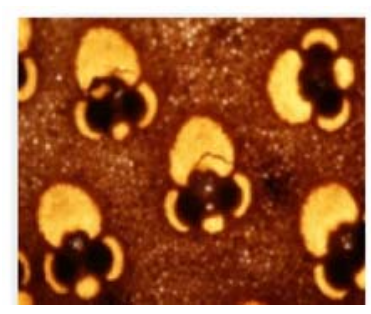

Región 1 (interna)

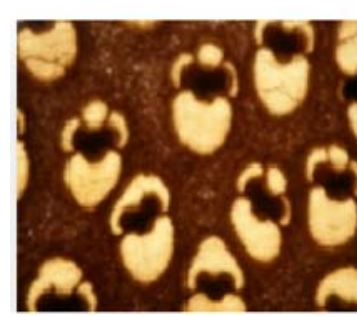

Región 2

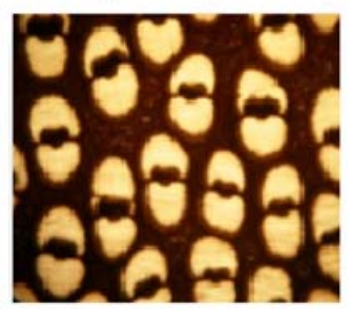

Región 3

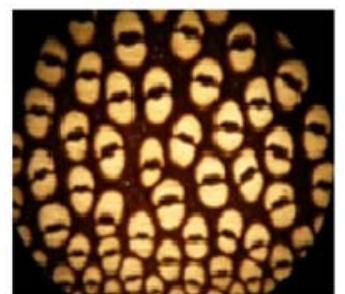

Región 4 (externa)

Figura 4: Microfotografías tomadas a las 4 regiones radiales consideradas en este trabajo. La zona de color café oscuro es el tejido parénquima, rodea a las fibras que poseen un tono claro; a su vez, los haces vasculares están rodeados por fibras. Las microfotografías fueron tomadas en el tramo correspondiente a la basa de la guadua etiquetada con el número 14.

En la Figura 4, se muestran las microfotografías tomadas a las 4 regiones radiales consideradas en el estudio. Se observa que el porcentaje de área ocupada por fibras cerca de la corteza aumenta en comparación con lo que se tiene en las otras regiones. Esta alta concentración de fibras causa aumento en la densidad $(\rho)$ [13]. Aunque los valores reportados en la Tabla 2 explican parcialmente la homogeneidad en los valores de DB y de difusividad térmica a lo largo de la guadua, estos no logran hacerlo para este mismo comportamiento de $\alpha$ en la dirección radial. Es posible que la composición química en estas regiones transversales sea la causa.

\section{CONCLUSIONES}

La guadua presenta valores de difusividad térmica alrededor de $(0,11 \pm 0,01) \times 10^{-6} \mathrm{~m}^{2} \mathrm{~s}^{-1}$ a lo largo del culmo. Este comportamiento está relacionado con la distribución de haces vasculares y parénquima. De igual manera, la dureza Brinell medida en la superficie externa o epidermis de la guadua no depende de la zona de muestreo cepa, basa o sobrebasa.

La difusividad térmica se mantiene prácticamente constante en la dirección radial para las regiones consideradas en la sección transversal de la guadua. Este comportamiento está en desacuerdo con el correspondiente a los parámetros morfoanatómicos estudiados en este trabajo, que fueron cuantificados como porcentaje de área ocupado por HV, F y P. Posiblemente, la composición química de la guadua permita entender tal situación y es en lo que se está trabajando actualmente.

\section{AGRADECIMIENTOS}

Este trabajo fue financiado por COLCIENCIAS y la Universidad del Quindío bajo el proyecto código 431.

\section{BIBLIOGRAFIA}

[1] MORA RODRÍGUEZ, L., HERNANDEZ GONZALES, J.F., et al., "El tablero de bambú prensado, una nueva propuesta tecnológica", Revista Ingeniería de la Construcción, v. 23, n. 3, pp. 179-186, diciembre 2008.

[2] GONZÁLEZ, H.Á., HELLWIG, S., MONTOYA, J. A., "Comportamiento a la cizalladura de vigas encoladas laminadas de guadua angustifolia kunth”, Scientia et Technica, v. 14, n. 39, pp. 428-433, septiembre 2008. 
[3] GONZÁLEZ, H.Á., HELLWIG, S., MONTOYA, J. A., "Determinación del adhesivo óptimo en la fabricación de vigas de bambú (guadua angustifolia)", Facultad de Ciencias Agropecuarias, v. 7, n. 1, pp. 67-75, mayo 2009.

[4] CRUZ RIOS, H., BAMBU - GUADUA: guadua angustifolia kunth, bosques naturales en colombia y plantaciones comerciales en México, 1 ed., Pereira Agromod, COLMEX, 2009.

[5] KANE, J.W., STERNHEIM, M.M., Física, 2ª ed., Barcelona, Editorial Reverté, 2007.

[6] ALMOND, D. P., PATEL, P.M., Photothermal science and techniques, $2^{\mathrm{a}}$ ed., New York, Chapman \& Hall, 1996.

[7] ROSENCWAIG. A., GERSHO. A., "Theory of the photoacoustic effect with solids", Journal. of Applied Physics, v. 47, n. 1, pp. 64-69, 1976.

[8] MONTOYA, J.A., GONZALES, H.A., BEDOYA, J.R., "Dureza Brinnel y la influencia de la humedad relativa del ambiente, de la edad y la altura a lo largo del tramo en la especie de bambú guadua angustifoliakunth", Scientia et Technica, v. 13, n. 34, pp. 619-624, maio 2007.

[9] MURILLO, D., ÁVILA, K., NARVÁEZ, C.A., "Simulación del ensayo de dureza Brinell para la aleación de aluminio 2024-T4 empleando el método de elementos finitos", Prospectiva, v. 7, n. 2, pp. 75-81, dezembro 2009.

[10] GROOVER, M.P., Fundamentos de manufactura moderna: materiales, procesos y sistemas, $1^{\mathrm{a}}$ ed., Mexico, Pearson Prentice Hall, 1997.

[11] VELEVA, L., TOMÁS, S.A., MARÍN, E., et al., "On the use of the photoacoustic technique for corrosion monitoring of metals: $\mathrm{Cu}$ and $\mathrm{Zn}$ oxides formed in tropical environments", Corrosion Science, v. 39, n. 9, pp. 1641-1655, setembro 1997.

[12] GORDILLO DELGADO, F., ORTEGA VELA, J. A., ZARATE RINCÓN, F., “Correlación entre parámetros térmicos de café de diferente origen”, Revista Colombiana de Física, v. 41, n. 1, pp. 168-170, janeiro 2009.

[13] GNANAHARAN, R., JANSSEN, J.J.A., "Bending strength of guadua bamboo: comparison of different testing procedures”, In: INBAR Working Paper No. 3, 1994. 